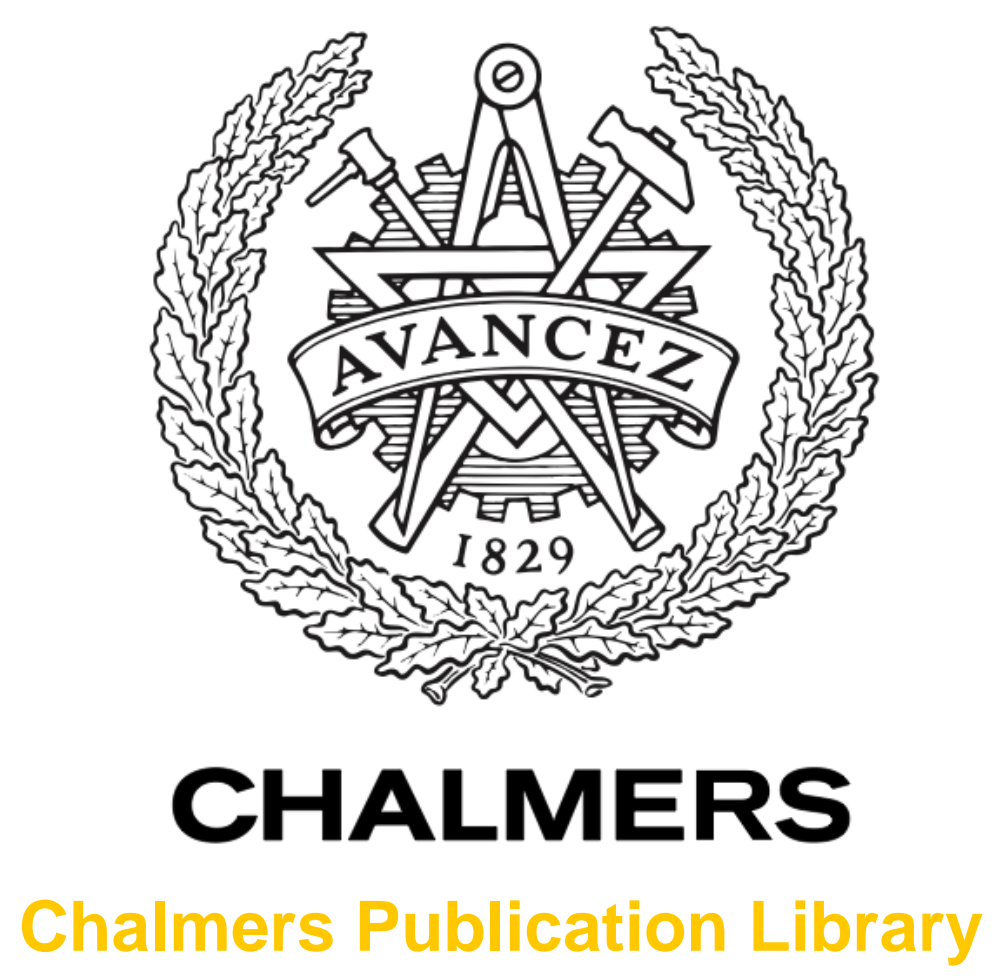

Spectral coherence in microresonator combs

This document has been downloaded from Chalmers Publication Library $(\mathrm{CPL})$. It is the author's version of a work that was accepted for publication in:

Conference on Lasers and Electro Optics 2014

Citation for the published paper:

Torres Company, V. ; Castello-Lurbe, D. ; Silvestre, E. (2014) "Spectral coherence in microresonator combs". Conference on Lasers and Electro Optics 2014

Downloaded from: http://publications.lib.chalmers.se/publication/201116

Notice: Changes introduced as a result of publishing processes such as copy-editing and formatting may not be reflected in this document. For a definitive version of this work, please refer to the published source. Please note that access to the published version might require a subscription.

Chalmers Publication Library (CPL) offers the possibility of retrieving research publications produced at Chalmers University of Technology. It covers all types of publications: articles, dissertations, licentiate theses, masters theses, conference papers, reports etc. Since 2006 it is the official tool for Chalmers official publication statistics. To ensure that Chalmers research results are disseminated as widely as possible, an Open Access Policy has been adopted.

The CPL service is administrated and maintained by Chalmers Library. 


\title{
Spectral Coherence in Microresonator Combs
}

\author{
Victor Torres-Company ${ }^{1, *}$, David Castelló-Lurbe ${ }^{2}$ and Enrique Silvestre ${ }^{2}$ \\ 1. Microtechnology and Nanoscience department, Chalmers University of Technology, 41321 Göteborg, Sweden \\ 2. Departamento d'Òptica, Universitat de València, 46000 Burjassot, Spain \\ torresv@chalmers.se
}

\begin{abstract}
We provide a quantitative analysis of the coherence in microresonator frequency combs. We show how to achieve coherent transform-limited pulses on-chip without actively manipulating the pump setting conditions in the course of comb formation.

OCIS codes: (320.7110) Ultrafast nonlinear optics; (030.1640) Coherence; (130.3990) Micro-optical devices.
\end{abstract}

\section{Introduction}

Microresonator combs exploit parametric oscillation and nonlinear mixing in an ultrahigh- $Q$ cavity pumped by an external continuous-wave (CW) laser [1]. The first demonstrations used whispering-gallery-mode resonators made of crystalline materials. Later, microresonator combs were demonstrated using planar geometries compatible with CMOS fabrication standards [2]. This new comb platform has got significant attention in the past few years owing to its unique potential for chip integration and access to ultra-high repetition rates [3]. Obtaining coherent broadband spectra is crucial to most of the applications foreseen with microresonator combs (such as optical and RF waveform generation) [3]. However, recent line-by-line pulse shaping [4] and multi-heterodyne beating [5] experiments indicate that there is an intricate spectral coherence behavior in the formation of microresonator combs.

The essential physics of these combs can be described using a modified nonlinear Schrödinger equation [6]. This treatment predicts that spectrally coherent combs can be obtained either at the onset of modulation instability (MI) or when temporal cavity solitons are formed [7]. Indeed, high-performance combs working in either regime have been reported in $[4,8]$. However, at the MI onset the comb may not lead to transform-limited pulses [4] and the formation of temporal cavity solitons requires very precise and active tuning of the pump laser characteristics [8]. Here, we provide a design rule that allows for obtaining coherent transform-limited pulses on chip without pump manipulation. The performance is robust to the noise conditions that initiate the comb dynamics.

\section{Results}

(a) $\delta_{0}=-0.014$

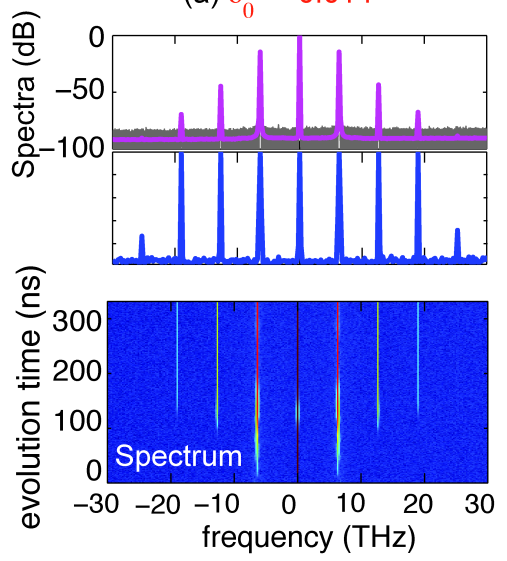

(b) $\delta_{0}=-0.007$
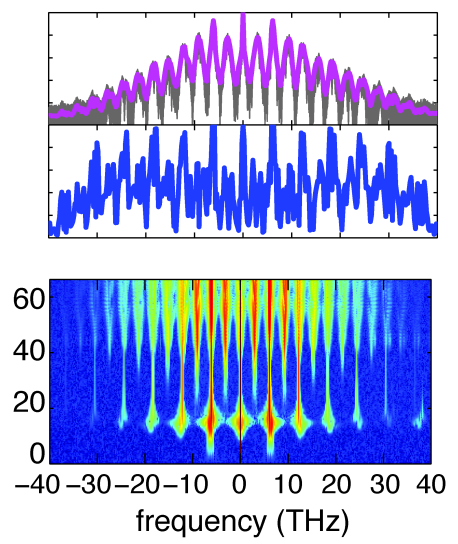

(c) $\delta_{0}=0$

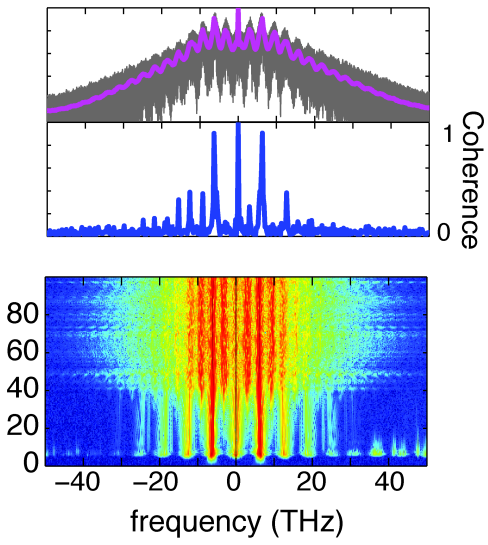

Fig. 1. Envelope comb spectra for different detuning conditions. Once the comb approaches the steady state, the degree of coherence is calculated [7]. Different spectral snapshots are overlaid (gray curves above) and the average is displayed in purple. The evolution of the comb over time (bottom figures) is calculated from the equation in [6].

Concretely, we show that when the group velocity dispersion, $\beta_{2}$ of the microring cavity satisfies

$$
\beta_{2}=\frac{\left(\delta_{0}-2 \gamma L P_{0}\right)}{2 L \pi^{2} F S R^{2}}
$$

the comb naturally evolves towards a coherent state providing ultrashort transform-limited pulses without the need for actively tuning the pump setting conditions. $\delta_{0}$ is the normalized detuning of the $\mathrm{CW}$ pump frequency, $\omega_{p}$, with 
respect to the closest cavity resonance, $\omega_{0}$, i.e. $\delta_{0}=\left(\omega_{0}-\omega_{p}\right) / F S R ; \gamma$ the nonlinear coefficient; $L$ the cavity length; FSR the free spectral range and $P_{0}$ relates to the CW pump power, $P_{i n}$, through $P_{0}\left[\alpha^{2}+\left(\delta_{0}-\gamma L P_{0}\right)^{2}\right]=\alpha P_{i n}$, where $\alpha$ denotes the total loss per roundtrip and we have assumed critical coupling. Equation (1) is obtained from imposing that the first oscillating comb line coincides with the cavity resonance adjacent to the pump. It can be understood as a generalization for the condition for coherent comb generation posed in [5], since $P_{0}$ is not necessarily the intracavity power at threshold. We also note that the detuning makes it possible to achieve coherent combs in the normal dispersion regime, as experimentally verified in e.g. [9].

We first characterize the spectral coherence properties of a microresonator comb with design parameters similar to the one reported in [6]. We consider a $\mathrm{CW}$ pump power of $1.5 \mathrm{~W}$. As Fig. 1 indicates, as the pump frequency gets closer into resonance $\left(\delta_{0} \approx 0\right)$, a broader comb is achieved since more power circulates into the cavity, but this process comes at the expense of a loss in spectral coherence. As expected [7], the comb is only coherent at the onset of MI [Fig.1 (a)]. We note that the first oscillating lines appear several FSRs away from the pump, i.e. the dispersion properties of the ring do not satisfy Eq. (1). This microresonator can provide coherent cavity solitons [7] but in practice this requires active and precise manipulation of the pump characteristics [8].
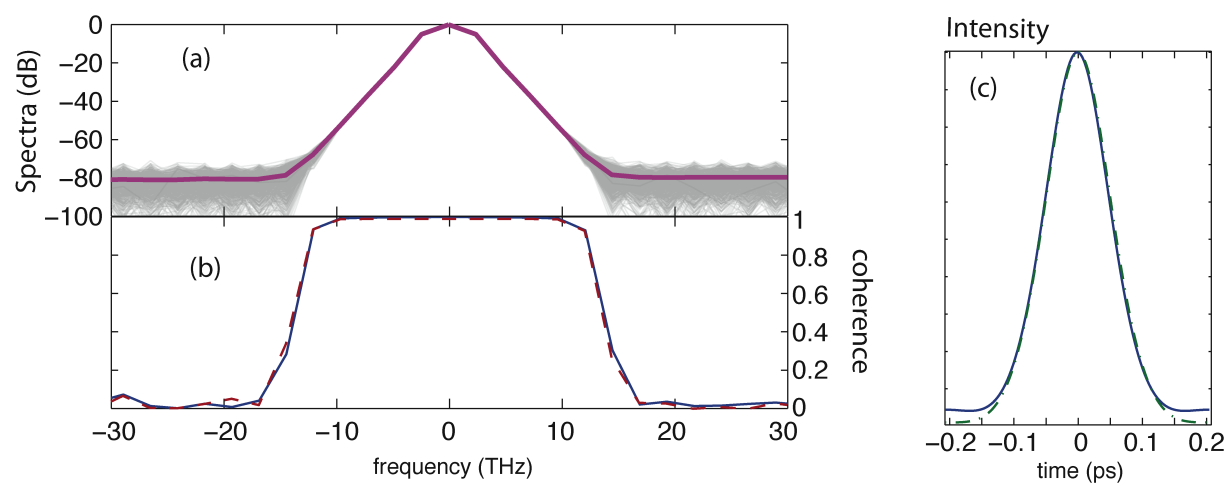

Fig. 2. (a) Snapshots of envelope comb spectra at different instant times (overlaid) and average spectra (purple solid curve). (b) The degree of coherence is high when calculated as in [7] (blue curve) as well as when calculated for different noise seeds [10]. (c) The time domain profile (blue curve) corresponds to a transform-limited pulse (green dashed line).

An alternative to achieve a broadband and spectrally coherent comb is to design the microresonator properties according to Eq. (1). Concretely, we assume on resonance pumping and critical coupling; $F S R=2.41 \mathrm{THz}$; $\alpha=0.001$ and $\gamma=1100(\mathrm{~W} \cdot \mathrm{km})^{-1}$, and the CW pump power is $0.2 \mathrm{~W}$. These parameters are experimentally feasible for silicon nitride microresonators [2]. The results are shown in Fig. 2. The comb envelope [Fig. 2 (a)] is narrower as compared to the case in Fig. 1 (c) because now the dispersion is higher. However the degree of coherence is superior [Fig. 2(b)]. Furthermore, the evolution towards the steady state now becomes extremely robust to the noise conditions that initiate the comb generation process. This can be seen in Fig. 2(b) (red dashed line) where the coherence is calculated over statistically independent noise seeds, similarly to what is done for assessing the coherence in supercontinuum sources [10]. More importantly, the steady state leads to an ultrashort pulse (blue line in Fig. 2(c), which is very close to the transform-limited duration (green dashed line).

In conclusion, we have analyzed the spectral degree of coherence in microresonator frequency combs. A design rule is provided that leads to broadband coherent transform-limited pulses. We have verified that the path towards this steady state does not require active pump manipulation and is indeed robust to the noise conditions that initiate the comb generation process.

\section{References}

[1] P. Del'Haye et al., "Optical frequency comb generation from a monolithic microresonator," Nature 450, 1214 (2007).

[2] D. J. Moss et al., "New CMOS-compatible platforms based on silicon nitride and Hydex for nonlinear optics," Nature Photon. 7, 597 (2013).

[3] T. J. Kippenberg, R. Holzwarth, and S. A. Diddams, "Microresonator-based optical frequency combs," Science 332, 555 (2011).

[4] F. Ferdous et al., "Spectral line-by-line pulse shaping of on-chip microresonator frequency combs," Nature Photon. 5, 770 (2011).

[5] T. Herr et al., "Universal formation dynamics and noise of Kerr-frequency combs in microresonators," Nature Photon. 6, 480 (2012).

[6] S. Coen et al., "Modeling of octave-spanning Kerr frequency combs using...," Opt. Lett. 38, 37 (2013).

[7] M. Erkintalo and S. Coen, "Coherence properties of Kerr frequency combs", Opt. Lett. 39, 283 (2014).

[8] T. Herr et al., "Temporal solitons in optical microresonators" doi:10.1038/nphoton.2013.343

[9] P. H. Wan et al., "Observation of correlation between route to formation, coherence, noise, and ..." Opt. Express 20, 29284 (2012).

[10] J. M. Dudley, G. Genty, and S. Coen, "Supercontinuum generation in photonic crystal fibers,” Rev. Mod. Phys. 78, 1135-1184 (2006). 